\title{
Inhibitory effect of the anthelmintic drug pyrvinium pamoate on T315I BCR-ABL-positive CML cells
}

\author{
JING ZHANG $^{1}$, YANLI JIN ${ }^{1}$ and JINGXUAN PAN ${ }^{1,2}$ \\ ${ }^{1}$ Jinan University Institute of Tumor Pharmacology, College of Pharmacy, Jinan University, Guangzhou, \\ Guangdong 510632; ${ }^{2}$ State Key Laboratory of Ophthalmology, Zhongshan Ophthalmic Center, \\ Sun Yat-Sen University, Guangzhou, Guangdong 510060, P.R. China
}

Received December 6, 2016; Accepted July 11, 2017

DOI: $10.3892 / \mathrm{mmr} .2017 .7685$

\begin{abstract}
Chronic myeloid leukemia (CML) is a clonal myeloproliferative disorder characterized by a chromosome translocation that generates the BCR-ABL oncogene, which encodes a constitutively activated tyrosine kinase. Despite progress in controlling CML at the chronic phase by first and second generations of BCR-ABL tyrosine kinase inhibitors (TKIs), effective drugs with good safety are not available for CML patients harboring T315I BCR-ABL and those in advanced stages of CML. Therefore, there is an urgent requirement for the development of effective therapies against T315I BCR-ABL. In the present study, it was demonstrated that pyrvinium pamoate, an anthelmintic drug approved by the Food and Drug Administration had potent inhibitory effects on growth and survival in CML cells with T315I BCR-ABL. In addition, this agent was equally effective in inhibiting the Wnt/ $\beta$-catenin signaling in wild-type and T315I BCR-ABL CML cells. Thus, the clinical efficacy of pyrvinium pamoate in treating patients with CML bearing T315I BCR-ABL should be further investigated.
\end{abstract}

\section{Introduction}

Chronic myeloid leukemia (CML) is a type of hematopoietic disorder arising from chromosomal abnormality between chromosomes 9 and 22 (1). This chromosomal abnormality, known as the Philadelphia chromosome, leads to myeloid

Correspondence to: Professor Jingxuan Pan or Dr Yanli Jin, Jinan University Institute of Tumor Pharmacology, College of Pharmacy, Jinan University, 601 West Huangpu Boulevard, Guangzhou, Guangdong 510632, P.R. China

E-mail: panjx2@mail.sysu.edu.cn

E-mail: yanlijin2014@163.com

Abbreviations: CML, chronic myeloid leukemia; TKIs, tyrosine kinase inhibitors; COX II, cytochrome $c$ oxidase subunit II; AIF, apoptosis-inducing factor

Key words: chronic myeloid leukemia, T315I, imatinib resistance, pyrvinium pamoate, apoptosis, $\beta$-catenin proliferation (2). Although tyrosine kinase inhibitor (TKI) imatinib mesylate (Gleevec) has been demonstrated to possess clinical benefit for patients with chronic phase CML harboring wild-type BCR-ABL, the CML patients harboring BCR-ABL mutants (e.g., E255V, Y253H, M351T and T315I) do not respond to imatinib (3). The majority of imatinib resistance is caused by BCR-ABL mutants, however other mutants can be overcome by the second-generation TKIs including dasatinib, nilotinib and bosutinib (4). For T315I mutation resistance to imatinib, effective therapeutics remain to be developed. The third-generation TKIs, such as ponatinib, show activity against CML with T315I BCR-ABL (5), however, the Food and Drug Administration (FDA) suspended its clinical use in 2013 due to its cardiovascular toxicity (6). Thus, agents effective against T315I BCR-ABL mutation would be valuable in treating refractory CML patients.

Pyrvinium(6-(dimethylamino)-2-[2-(2,5-dimethyl-1-phenylpyrrol-3-yl)ethenyl]-1-methyl-quinolinium) pamoate (Fig. 1A) is a FDA-approved drug used for the treatment of pinworm infections (7). Previous studies have demonstrated that pyrvinium pamoate can effectively inhibit the growth of tumor cells of diverse tissue origins, including the pancreas, colon, breast, brain, myeloma and erythroleukemia (8-14). It also has been demonstrated that pyrvinium pamoate exhibits increased cytotoxicity in tumor cells under hypoglycemic conditions and enhances the effects of conventional chemotherapeutic drugs in tumor xenografts $(10,12,15)$. A previous study reported that pyrvinium pamoate was active against CML patients harboring wild-type BCR-ABL (16). However, whether it is against T315I BCR-ABL CML cells remains to be elucidated.

In the present study, the activity of pyrvinium pamoate was determined in CML cells with T315I BCR-ABL. It was identified that pyrvinium pamoate potently suppressed proliferation and induced apoptosis, and inhibited the Wnt/ $\beta$-catenin signaling pathway in the CML cells with T315I BCR-ABL. These results suggest that pyrvinium pamoate may be an effective drug for the treatment of CML with T315I BCR-ABL mutation.

\section{Materials and methods}

Reagents and antibodies. Pyrvinium pamoate (CAS: 3546-41-6; chemical structure, Fig. 1A) was purchased 
from Sigma-Aldrich (Merck KGaA, Darmstadt, Germany) and was prepared as a $20 \mathrm{mM}$ stock solution in dimethyl sulfoxide (DMSO) and stored at $-20^{\circ} \mathrm{C}$. Antibodies and their sources were as follows: Mouse monoclonal antibodies against poly ADP-ribose polymerase (PARP; clone 4C10-5; cat no. 51-6639GR; dilution, 1:4,000), X-linked inhibitor of apoptosis protein (XIAP; cat no. 610762; dilution, 1:1,000), c-Myc (cat no. 51-1485GR; dilution, 1:1,000), $\beta$-catenin (cat no. 610154; dilution, 1:1,000), cytochrome $c$ (clone 6H2.B4; cat no. 556432; dilution, 1:1,000), caspase-3 (cat no. 610322; dilution, 1:1,000) and rabbit polyclonal antibodies against active caspase-3 (cat no. 557038; dilution, 1:500) were from BD Pharmingen (San Diego, CA, USA). Antibodies against B-cell lymphoma (Bcl)-2-associated X (Bax; cat no. sc-493; dilution, 1:1,000), Bcl- $\mathrm{X}_{\mathrm{L}}$ (S-18) (cat no. sc-634; dilution, 1:1,000), glycogen synthase kinase (GSK) $3 \beta$ (cat no. sc-9166; dilution, 1:1,000), cyclin D1 (cat no. sc-753; dilution, 1:1,000) and mouse monoclonal antibodies against apoptosis-inducing factor (AIF; cat no. sc-55519; dilution, 1:500), were from Santa Cruz Biotechnology, Inc. (Dallas, TX, USA); antibodies against $\beta$-actin (clone AC-15; cat no. A5441; dilution, 1:4,000) and $\alpha$-tubulin (clone B-5-1-2; cat no. T5168; dilution, 1:1,000) were from Sigma-Aldrich; Merck KGaA; antibodies against caspase-8 (cat no. 9746S; dilution, 1:1,000), caspase-9 (cat no. 9508S; dilution, 1:1,000), phosphorylated (p)-GSK3 $\beta$ (Ser9; cat no. 9336S; dilution, 1:1,000) and Mcl-1 (cat no. 4572S; dilution, 1:500) were from Cell Signaling Technology, Inc. (Danvers, MA, USA). Anti-survivin (cat no. NB 500-201; dilution, 1:1,000) was from Novus Biologicals, Bcl-2 (cat no. 05-729; dilution, 1:1,000) was from EMD Millipore (Billerica, MA, USA) and cytochrome $c$ oxidase subunit II (COX II; dilution, 1:1,000) was purchased from Invitrogen (Thermo Fisher Scientific, Inc., Waltham, MA, USA). The secondary Alexa Fluor ${ }^{\circledR} 680 \mathrm{CW}$ goat anti-mouse (cat no. 926-68070; dilution, $1: 10,000$ ) and $800 \mathrm{CW}$ goat anti-rabbit (cat no. 926-32211; dilution, 1:10,000) antibodies were purchased from LI-COR Biosciences (Lincoln, NE, USA).

Cell culture. K562 cells were purchased from American Type Culture Collection (Manassas, VA, USA), and grown in RPMI-1640 (Invitrogen; Thermo Fisher Scientific, Inc.) with $10 \%$ heat inactivated fetal bovine serum (FBS; Biological Industries USA, Cromwell, CT, USA), maintained in a humidified atmosphere with $5 \% \mathrm{CO}_{2}$ at $37^{\circ} \mathrm{C}$. $\mathrm{KBM} 5$ and KBM5-T315I cells were provided by Professor Sai-Ching J. Yeung (Department of Emergency Medicine, University of Texas MD Anderson Cancer Center, Houston, TX, USA) and grown in Iscove's modified Dulbecco's medium (Invitrogen; Thermo Fisher Scientific, Inc.) supplemented with $10 \%$ heat inactivated FBS as previously described (17). The 32D myeloid cells stably expressing either $210 \mathrm{kDa}$ wild-type BCR-ABL (32D-BCR-ABL) or T315I BCR-ABL [32D-T315I; both provided by Dr Lin Qiu (Harbin Institute of Hematology and Oncology, Harbin, Heilongjiang, China)] were established and maintained in RPMI-1640 with 10\% FBS as described previously $(18,19)$.

Cell viability assay. Cell viability was determined by MTS assay (CellTiter 96 Aqueous One Solution Cell Proliferation assay; Promega Corporation, Madison, WI, USA) (20). Briefly,
$2 \times 10^{4}$ cells in $100 \mu 1$ were exposed to various concentrations of pyrvinium pamoate for $72 \mathrm{~h}$. Control cells were treated with medium containing $<0.1 \%$ DMSO. The absorbance/optical density were read on a 96 -well plate reader at wave length $490 \mathrm{~nm}$. The drug concentration resulting in 50\% inhibition of cell growth $\left(\mathrm{IC}_{50}\right)$ was determined (21).

Colony formation assays. The K562, KBM5, KBM5-T315I, 32D-BCR-ABL and 32D-T315I cells were treated with pyrvinium pamoate or DMSO (control) for $24 \mathrm{~h}$, then cells were harvested and washed with PBS, and 2,000 cells were seeded in RPMI-1640 or Iscove's medium containing $0.3 \%$ agar and $20 \%$ FBS in the absence of drug treatment. Following incubation for another $10 \sim 14$ days at $37^{\circ} \mathrm{C}$ and $5 \% \mathrm{CO}_{2}$, colonies with $>50$ cells were counted using an inverted phase-contrast microscope (22).

Trypan blue exclusion assay. The K562, KBM5, KBM5-T315I, 32D-BCR-ABL and 32D-T315I cells were cultured at $2 \times 10^{5}$ cells/well in 6 -well plates, then cells were treated with increasing concentrations of pyrvinium pamoate, or with the fixed concentration for different time periods. Viable and dead cells were assessed by counting with a hemocytometer following trypan blue exclusion assay (23).

Preparation of cell lysates and cytosolic fractions. Whole lysates were prepared with radioimmunoprecipitation assay buffer (1X PBS, $1 \%$ NP-40, $0.5 \%$ sodium deoxycholate, $0.1 \%$ SDS) supplemented with freshly added $10 \mathrm{mM} \beta$-glycerophosphate, $1 \mathrm{mM}$ sodium orthovanadate, $10 \mathrm{mM} \mathrm{NaF}, 1 \mathrm{mM}$ phenylmethylsulfonyl fluoride, and Roche Complete Mini Protease Inhibitor Cocktail (Roche Diagnostics, Basel, Switzerland) (24). The cytosolic fraction was prepared with digitonin extraction buffer to detect the level of cytochrome $c$ and AIF in the cytosol, as described previously (25).

Preparation of cytoplasmic and nuclear fractions. Control or drug-treated cells were pelleted by centrifugation at $300 \mathrm{x} \mathrm{g}$ for $5 \mathrm{~min}$ at room temperature and rinsed with PBS. Pellets were then resuspended in $200 \mu \mathrm{l}$ ice-cold lysis buffer $(10 \mathrm{mM}$ Hepes pH 7.9, $10 \mathrm{mM} \mathrm{KCl,} 0.1 \mathrm{mM}$ EDTA, 0.4\% NP-40 with $1 \mathrm{mM}$ DTT, $0.5 \mathrm{mM}$ PMSF, $1 \mathrm{mM}$ sodium orthovanadate and the Roche Complete Mini Protease Inhibitor Cocktail) by pipetting up and down (without bubbling) 10 times $(22,26)$. Following incubation on ice for $10 \mathrm{~min}$, the lysates were centrifuged at $10,000 \mathrm{x}$ g for $10 \mathrm{~min}$ at $4^{\circ} \mathrm{C}$. The supernatants were transferred to fresh tubes and referred to as cytoplasm ice extracts. Following washing with the lysis buffer, the pellets were resuspended in nuclear protein extraction buffer with inhibitors (20 mM HEPES, pH 7.9, 0.4 M NaCl, 1 mM EDTA with $1 \mathrm{mM}$ DTT, $0.5 \mathrm{mM}$ PMSF, $0.2 \mathrm{mM}$ sodium orthovanadate and the Roche Complete Mini Protease Inhibitor Cocktail) and centrifuged at $10,000 \mathrm{xg}$ for $10 \mathrm{~min}$ at $4^{\circ} \mathrm{C}$. The resultant supernatants were kept as nuclear fractions $(22,26)$.

Western blotting analysis. The whole cells were lysed in radioimmunoprecipitation assay buffer on ice for $30 \mathrm{~min}$. Equal volumes $(20 \mu \mathrm{l})$ of lysate were electrophoresed with $10 \%$ 
A

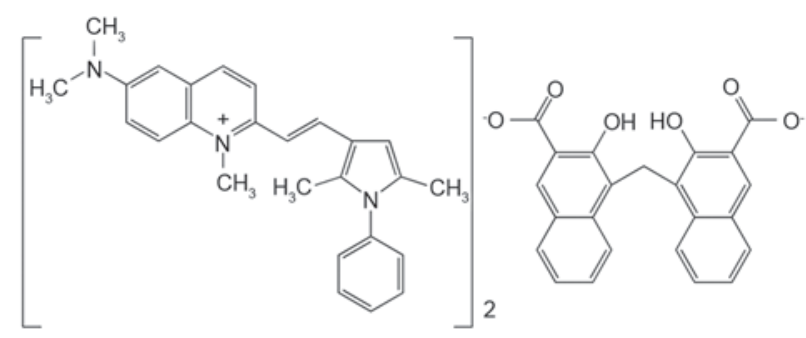

Pyrvinium pamoate

B

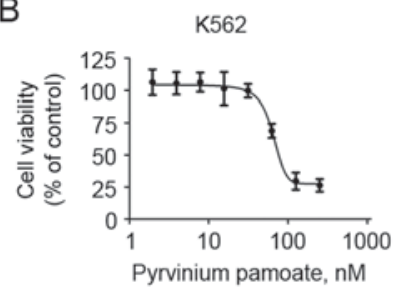

C

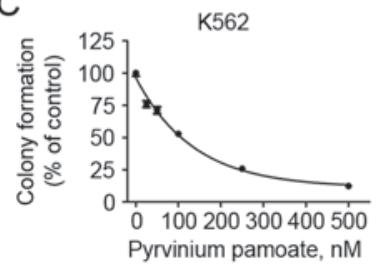

KBM5

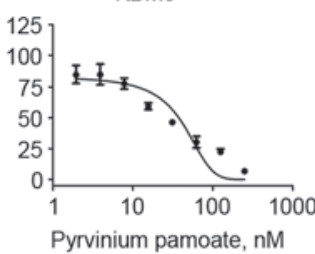

KBM5

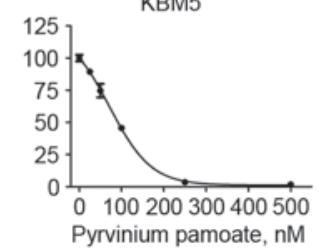

KBM5-T315I

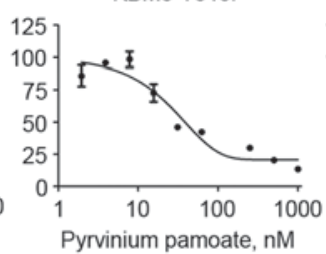

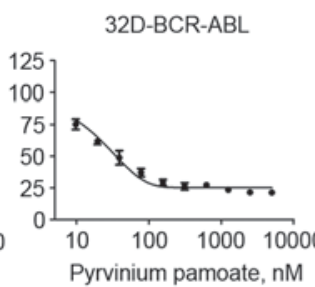

Pyrvinium pamoate, $\mathrm{nM}$

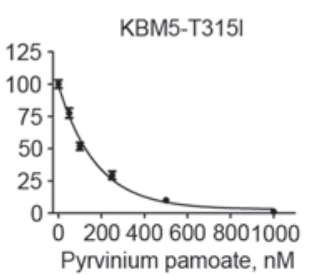

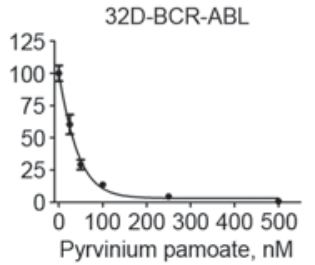

32D-T315।

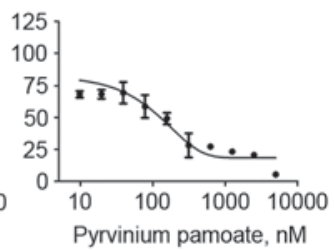

32D-T315I

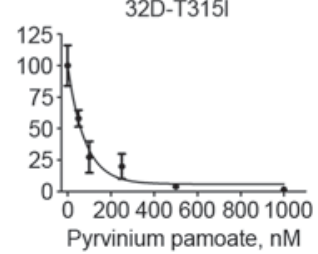

Figure 1. Pyrvinium pamoate reduced the growth of CML cells with T315I or wild-type BCR-ABL. (A) The chemical structure of pyrvinium pamoate. (B) Five lines of BCR-ABL-expressing cells were cultured with escalating concentrations of pyrvinium pamoate for $72 \mathrm{~h}$, followed by MTS assay to test the cell viability. Log dose-response curves with mean \pm standard deviation are presented. (C) Pyrvinium pamoate inhibited the clonogenicity of five lines of BCR-ABL-expressing cells in a dose-dependent manner. CML cells were treated with pyrvinium pamoate at the indicated concentrations for $24 \mathrm{~h}$ and then seeded in the drug-free soft agar for another 10-14 days of incubation, colony-forming units were counted. CML, chronic myeloid leukemia.

SDS-polyacrylamide gels and electrotransferred to nitrocellulose membranes. The membranes were blocked in PBS with $0.05 \%$ Tween-20 containing $5 \%$ skimmed milk and incubated with the primary antibody at $4^{\circ} \mathrm{C}$ overnight, and then incubated with the secondary 680/800 antibodies. Protein expression was assessed by the Odyssey infrared imaging system (LI-COR Biosciences). $\beta$-actin was used as a protein loading control.

Statistical analysis. All experiments were performed a minimum of three times, and data are presented as the mean \pm standard deviation. Differences between multiple groups were analyzed by one-way analysis of variance, with post-hoc intergroup comparisons made using Tukey's test, unless otherwise stated. GraphPad Prism 5.0 software (GraphPad Software, Inc., San Diego, CA, USA) was used for statistical analysis. $\mathrm{P}<0.05$ was considered to indicate a statistically significant difference.

\section{Results}

Pyrvinium pamoate effectively inhibited the proliferation of CML cells harboring T315I BCR-ABL. Firstly, the effect of pyrvinium pamoate on cell viability was examined. A total of three lines of human CML cells were incubated with increasing concentrations of pyrvinium pamoate (Fig. 1A) for $72 \mathrm{~h}$. The MTS assay indicated that K562 and KBM5 cells, both of which harbor wild-type BCR-ABL, were sensitive to pyrvinium pamoate with $\mathrm{IC}_{50}$ values of 14.2 and $26.2 \mathrm{nM}$, respectively (Fig. 1B), KBM5-T315I cells harboring T315I BCR-ABL exhibited a similar sensitivity to pyrvinium pamoate with an $\mathrm{IC}_{50}$ value $29.2 \mathrm{nM}$. The ability of pyrvinium pamoate to inhibit the viability was further confirmed in a pair of murine 32D myeloid cells stably transfected with either the wild-type or T315I BCR-ABL, the $\mathrm{IC}_{50}$ values were 49.7 and $133 \mathrm{nM}$, respectively (Fig. 1B). In an additional independent set of experiments, similar results were obtained in colony formation experiments, which indicated that pyrvinium pamoate inhibited the clonogenicity of CML cells bearing either wild-type BCR-ABL or T315I BCR-ABL (Fig. 1C).

These results demonstrated that pyrvinium pamoate could be equally effective in inhibiting the proliferation of $\mathrm{CML}$ cells with T315I BCR-ABL mutation as to that of wild-type CML cells.

Pyrvinium pamoate induced the apoptosis in CML cells harboring T315I BCR-ABL. Whether pyrvinium pamoate was able to induce cell death was investigated. The cells were cultured with increasing concentrations of pyrvinium pamoate for $36 \mathrm{~h}$, and cell death was measured by trypan blue exclusion assay. Pyrvinium pamoate led to marked increases in cell death in a dose- and time-dependent manner in the CML cells harboring either wild-type or T315I BCR-ABL (Fig. 2A and B). In addition, pyrvinium pamoate induced a dose- and time-dependent cleavage of PARP and caspase- 3 in all five lines of BCR-ABL-expressing cells (Fig. 2C and D). The levels of caspase-9 and -8 were also 

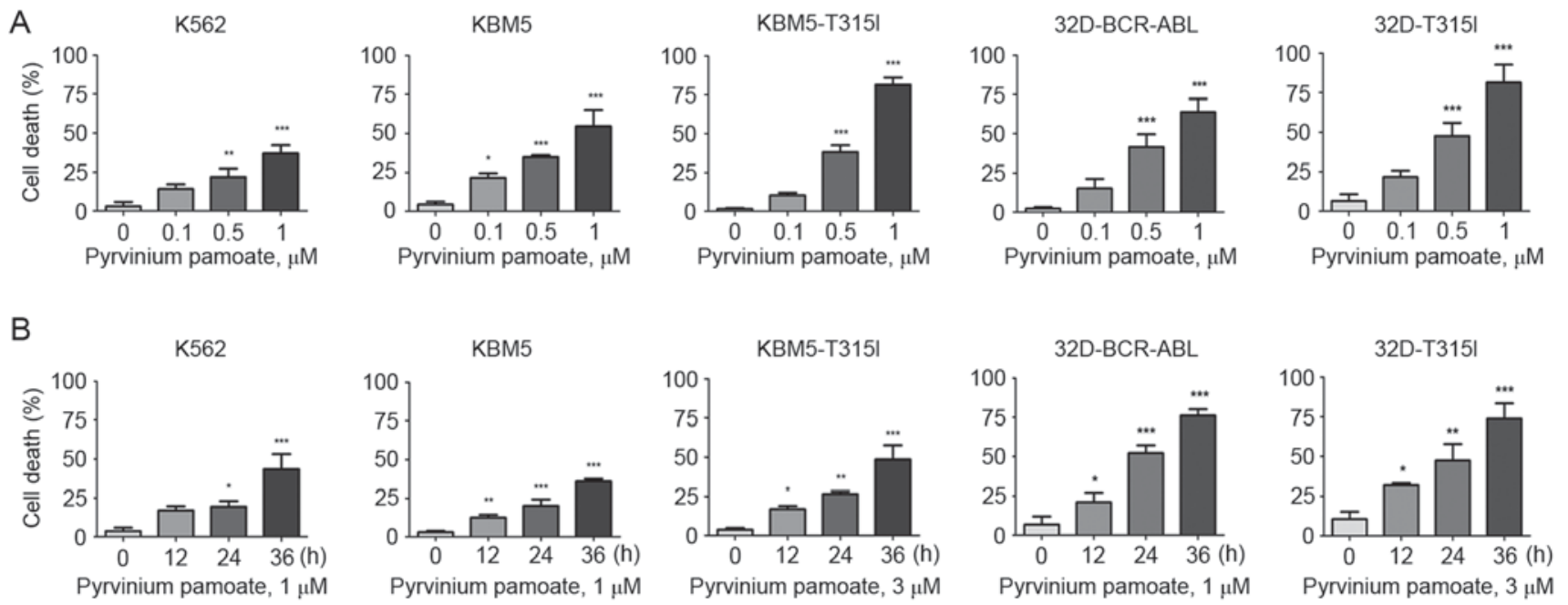

C

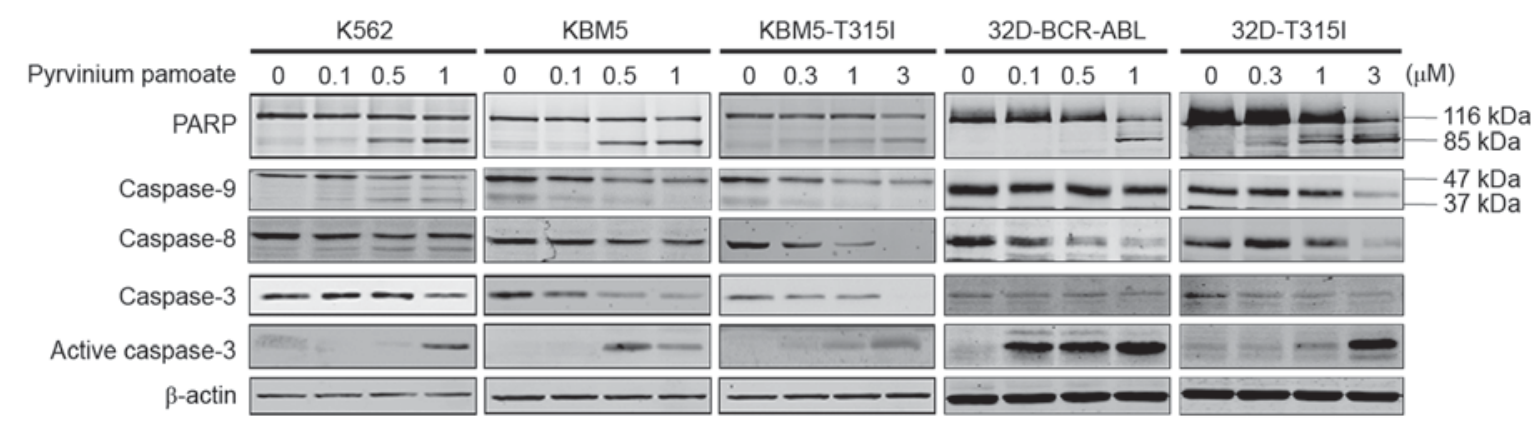

D

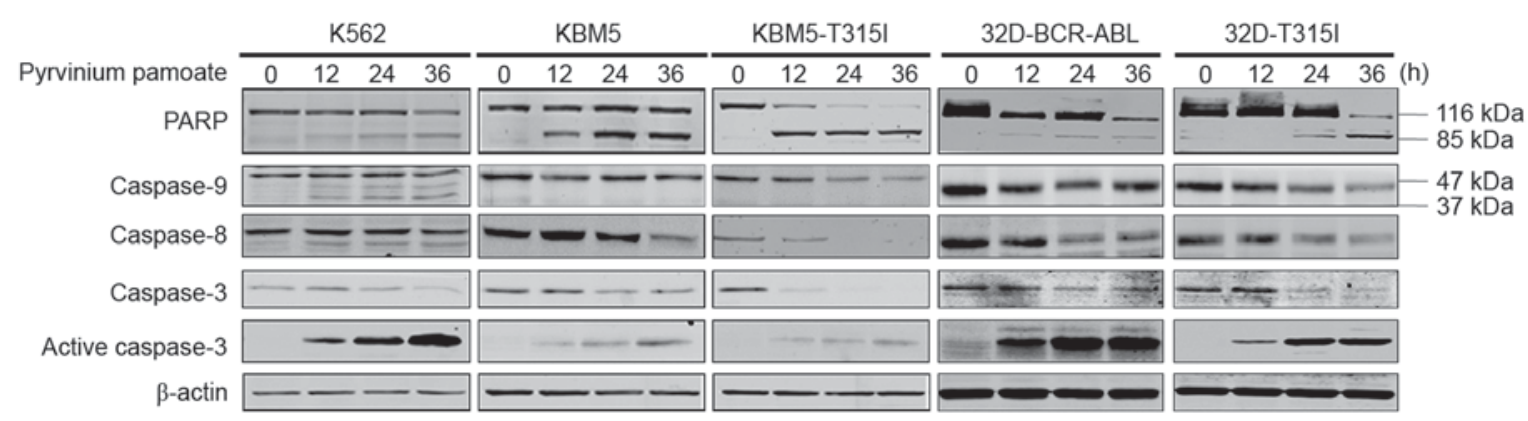

Figure 2. Pyrvinium pamoate induced apoptosis in CML cells with T315I BCR-ABL. (A and B) Cell death was evaluated by trypan blue exclusion assay. (A) K562, KBM5 and 32D-BCR-ABL cells were treated with pyrvinium pamoate $(0,0.1,0.5$ and $1 \mu \mathrm{M})$ for $36 \mathrm{~h}$, KBM5-T315I and 32D-T315I cells were treated with pyrvinium pamoate $(0,0.3,1$ and $3 \mu \mathrm{M})$ for $36 \mathrm{~h}$. (B) K562, KBM5 and 32D-BCR-ABL cells were treated with $1 \mu \mathrm{M}$ pyrvinium pamoate for 0 , 12, 24 and $36 \mathrm{~h}$; KBM5-T315I and 32D-T315I cells were treated with $3 \mu \mathrm{M}$ pyrvinium pamoate for $0,12,24$ and $36 \mathrm{~h}$. Trypan blue exclusion assay was used to measure the cell death of the five cell lines. ${ }^{*} \mathrm{P}<0.05,{ }^{* *} \mathrm{P}<0.01,{ }^{* * *} \mathrm{P}<0.001$, one-way analysis of variance with post hoc intergroup comparison by the Tukey's test. (C and D) Dose- and time-course western blotting analysis of PARP cleavage, caspase-3, -8 , and -9 in the whole-cell lysates of CML cells were shown. $\beta$-actin was used as a loading control. CML, chronic myeloid leukemia; PARP, poly adenosine diphosphate-ribose polymerase.

decreased in a dose- and time-dependent manner, suggesting their activation by pyrvinium pamoate (Fig. 2C and D). Pyrvinium pamoate additionally induced a time-dependent release of AIF and cytochrome $c$ into cytosol (Fig. 3A). Additionally, in all the drug-treated BCR-ABL-expressing cells, a decrease in the protein levels of anti-apoptotic proteins Mcl-1, survivin and XIAP was observed (Fig. 3B and C) but not of other Bcl-2 family members. Taken together, pyrvinium pamoate effectively induced apoptosis in five lines of BCR-ABL-expressing cells regardless of their BCR-ABL status.

Pyrvinium pamoate effectively inhibited the Wnt/ $\beta$-catenin signaling in CML cells harboring T315I BCR-ABL. It was investigated whether pyrvinium pamoate affected BCR-ABL expression, and western blotting analysis indicated no change in BCR-ABL expression in the CML cells treated with pyrvinium pamoate (Fig. 4A), suggesting that the antineoplastic effect of pyrvinium pamoate on CML cells is BCR-ABL independent. Previous reports have demonstrated that pyrvinium pamoate inhibits Wnt/ $\beta$-catenin signaling pathway in breast and lung cancer $(13,27)$. It was therefore investigated whether pyrvinium pamoate inhibited the Wnt $/ \beta$-catenin signaling in CML cells. The levels of $\beta$-catenin were markedly decreased in two pairs of CML cells (KBM5 and KBM5-T315I cells or 32D-BCR-ABL and 32D-T315I cells)(Fig. 4B). Subsequently, the upstream and downstream proteins of the $\mathrm{Wnt} / \beta$-catenin signaling pathway were 
A

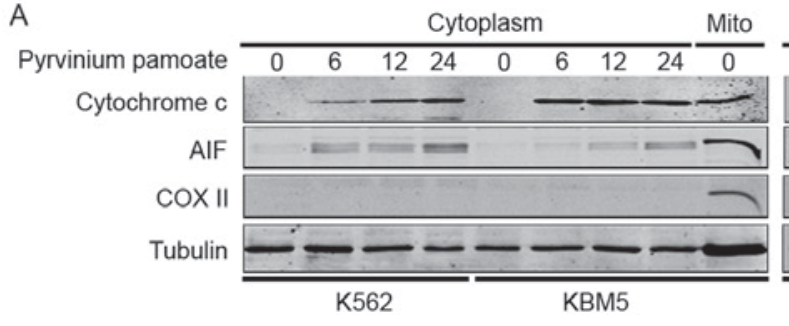

B

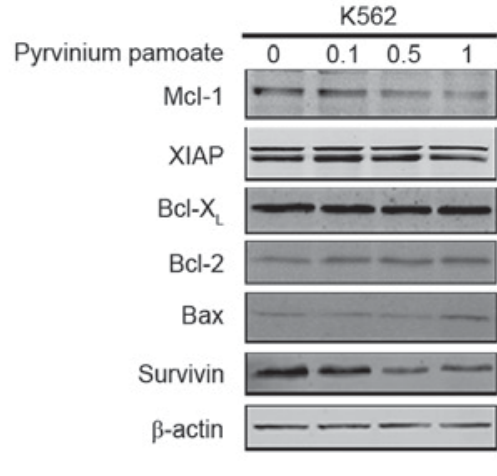

C

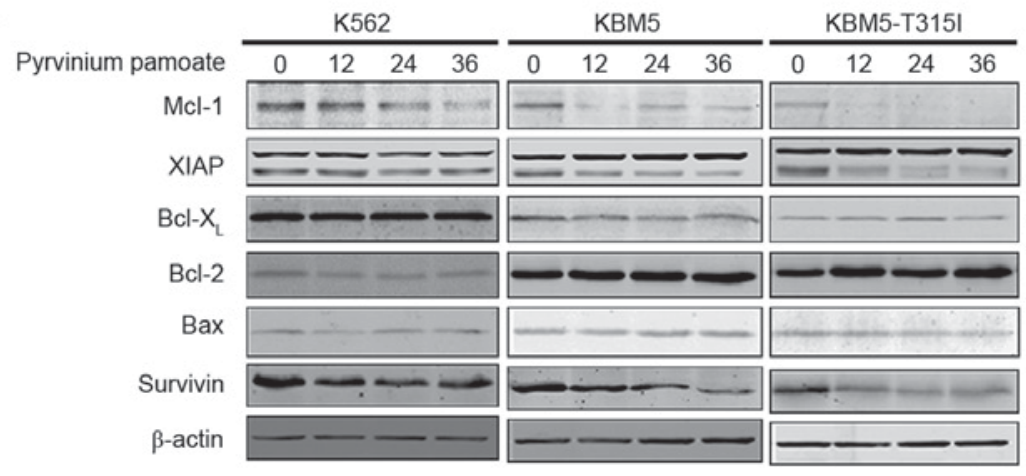

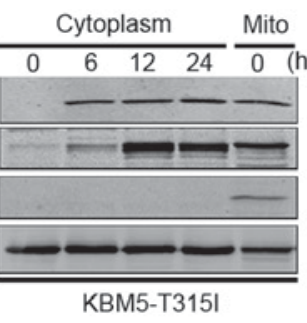

Pyrvinium pamoate

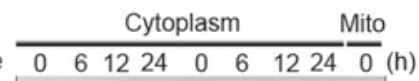

Cytochrome c

cox॥

Tubulin

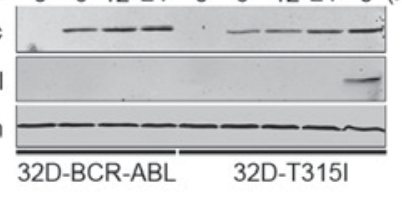

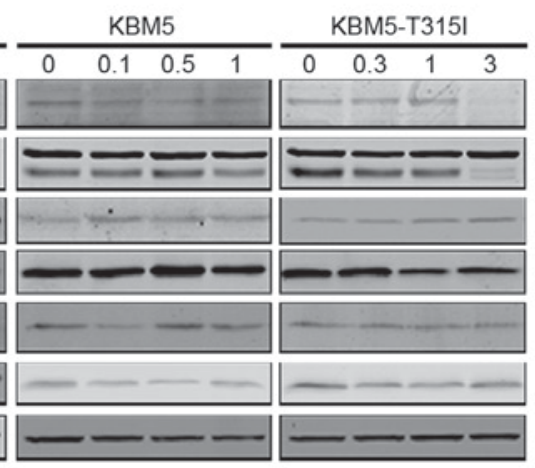

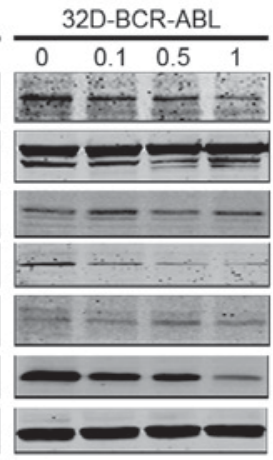

32D-BCR-ABL

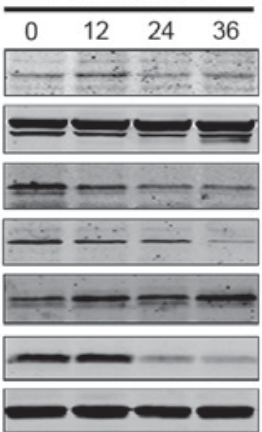

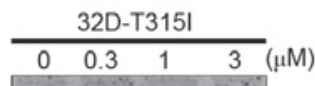

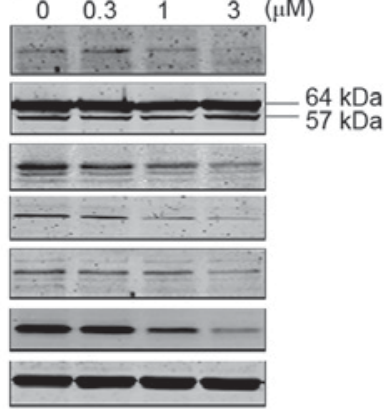

32D-T315!

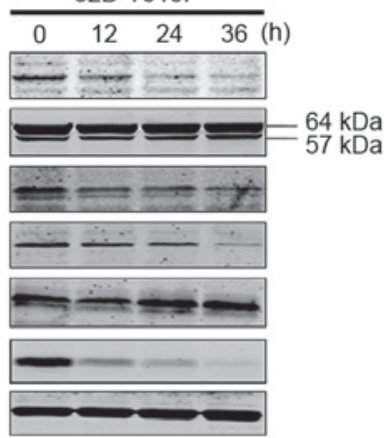

Figure 3. Pyrvinium pamoate regulated the expression of the apoptosis-associated proteins in CML cells with T315I BCR-ABL. (A) Pyrvinium pamoate induced release of AIF and cytochrome $c$ from the mitochondria. COX II served as a mitochondrial indicator. (B and C) The expression of apoptosis-associated proteins in whole cell lysates was detected by western blotting analysis. The cells were treated with (B) different concentrations of pyrvinium pamoate for $36 \mathrm{~h}$ or (C) $1 \mu \mathrm{M}$ pyrvinium pamoate for K562, KBM5 and 32D-BCR-ABL cells; or $3 \mu \mathrm{M}$ pyrvinium pamoate for KBM5-T315I and 32D-T315I cells for the indicated time periods. $\beta$-actin was used as a loading control. CML, chronic myeloid leukemia; AIF, apoptosis-inducing-factor; COX II, cytochrome $c$ oxidase subunit II; XIAP, X-linked inhibitor of apoptosis protein; Bcl, B-cell lymphoma; Bax, Bcl-2-associated X; Mcl-1, myeloid cell leukemia sequence 1.

measured. The results indicated that the levels of p-GSK3 $\beta$ (Ser9), cyclin D1 and c-Myc were decreased (Fig. 4B), suggesting that pyrvinium pamoate may block the activation of canonical Wnt/ $\beta$-catenin signaling.

Due to the fact that nuclear translocation of $\beta$-catenin is required for its functions (i.e., to activate T-cell factor/lymphoid enhancer factor), the effects of pyrvinium pamoate on the subcellular distribution of $\beta$-catenin were measured. The results indicated that the levels of $\beta$-catenin in nuclear and cytosolic fractions were reduced by pyrvinium pamoate in a dose-dependent manner (Fig. 4C).

\section{Discussion}

The present study aimed to investigate the anti-tumor activity of pyrvinium pamoate, an anthelminthic drug, in CML cells harboring the T315I mutation. The results demonstrated that this agent is equally effective in inhibiting proliferation and inducing apoptosis in CML cells harboring either wild-type or T315I BCR-ABL. Due to the fact that pyrvinium pamoate is an FDA-approved drug, the results of the present study may lead to a clinical trial for imatinib-resistant patients with T315I BCR-ABL.

Pyrvinium pamoate is a quinolone-derived cyanine dye, and the FDA approved its anthelmintic property in the 1950s (28). This agent has been previously investigated in solid tumors and hematopoietic malignancy (8-14). It was identified that pyrvinium pamoate potently inhibited the viability and induced apoptosis in both wild-type and T315I BCR-ABL-expressing cells at low nanomolar concentrations. The results were consistent with the report by Xiang et al (16) regarding the effect of pyrvinium pamoate on CML cells with wild-type BCR-ABL. Of note, pyrvinium pamoate was reported to be active against primary $\mathrm{CD} 34^{+}$blast cells from 8 patients with CML who harbored BCR-ABL point mutations (E255K, E453K and E255V). The results of the present study were in agreement with those from the literature (16), suggesting that this agent may be a promising potential therapy 
A

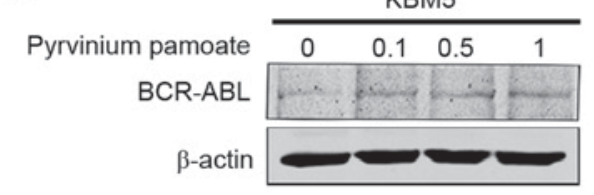

B

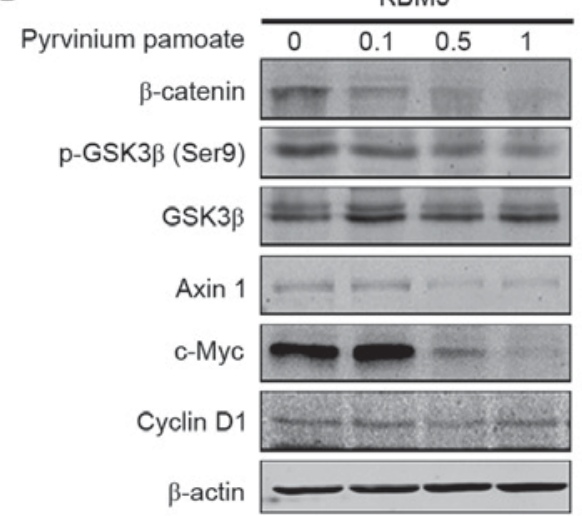

C

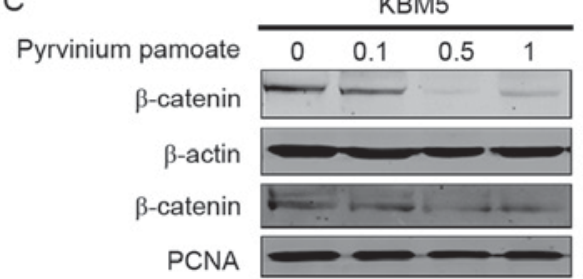

KBM5-T315I

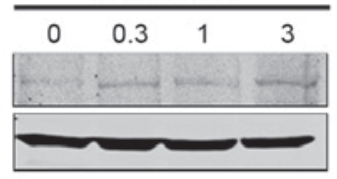

KBM5-T315I

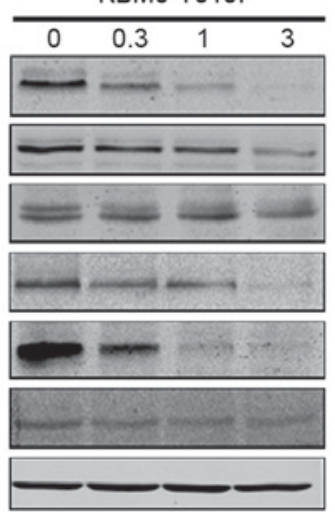

KBM5-T315।

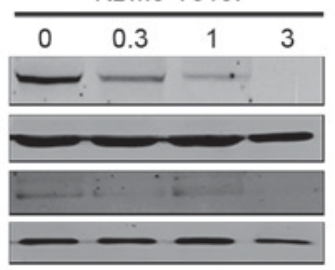

32D-BCR-ABL

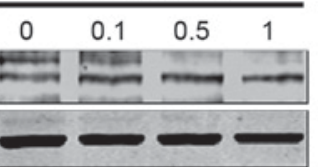

32D-BCR-ABL

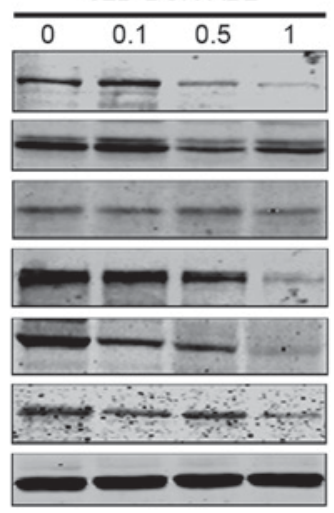

32D-BCR-ABL

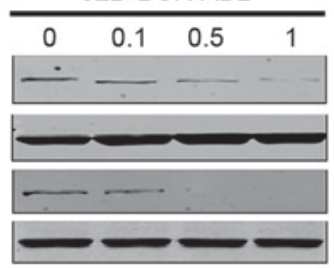

32D-T315।

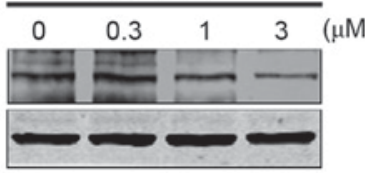

32D-T315।
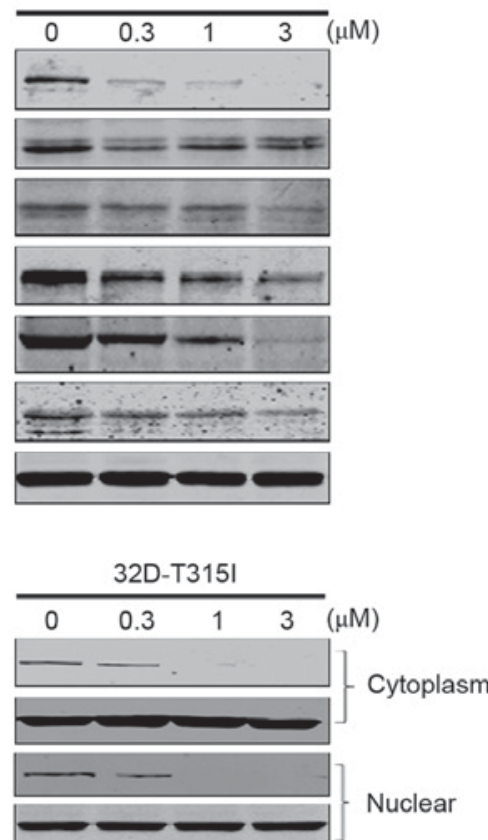

Figure 4. Pyrvinium pamoate inhibited Wnt/ $\beta$-catenin signaling in CML cells with T315I BCR-ABL. (A) Western blotting was performed to examine the levels of BCR-ABL in CML cells treated with pyrvinium pamoate. (B) CML cells were incubated with incremental dose of pyrvinium pamoate for $36 \mathrm{~h}$, cell lysates were subjected to western blotting analysis. (C) The levels of $\beta$-catenin in cytoplasm and nucleus were determined by western blotting analysis. CML, chronic myeloid leukemia; p-, phosphorylated; GSK, glycogen synthase kinase; PCNA, proliferating cell nuclear antigen.

for patients with CML who harbor wild-type and different point mutations of BCR-ABL including T315I.

Although it has been used for more than 50 years, the molecular mechanisms of pyrvinium pamoate remain unclear. It has been reported that in different types of cancer that pyrvinium pamoate may target different signaling pathways (8-11). We observed that the inhibitory effects of pyrvinium pamoate on mutant CML cells might be associated with its apoptotic action. Apoptosis, which is programmed cell death, can occur via the cell death receptor-mediated extrinsic pathway or the mitochondria-mediated intrinsic pathway (29). Reduction of apoptosis is believed to contribute to cancer development. In the present study, it was observed that pyrvinium pamoate induced apoptosis in both the wild-type and T315I BCR-ABL mutant CML cells (Figs. 2 and 3). The apoptosis may be caused by the mitochondrial-dependent pathway, as evidenced by the release of AIF and cytochrome $c$ to the cytosol was increased following treatment with pyrvinium pamoate (Fig. 3A).

Previous studies indicated that pyrvinium pamoate could inhibit the growth and differentiation of different cancer cells through the Hedgehog signaling pathway (14), or Wnt $/ \beta$-catenin signaling pathway $(13,27,30)$. Consistent with those results, decreased levels of $\beta$-catenin were observed, and the upstream and downstream proteins of $\mathrm{Wnt} / \beta$-catenin pathway, including phospho-GSK3 $\beta$ (Ser 9) and c-Myc in
CML cells either with wild-type or T315I BCR-ABL mutations following pyrvinium pamoate treatment (Fig. 4B). Thus, it is suggested that pyrvinium pamoate inhibits the growth of CML cells by inhibiting the Wnt/ $\beta$-catenin signaling pathway. Taken together, it may be beneficial to investigate the clinical efficacy of pyrvinium pamoate in treating patients with CML bearing T315I BCR-ABL.

In summary, it was reported that pyrvinium pamoate effectively inhibits the proliferation and induces apoptosis in the wild-type or T315I BCR-ABL-expressing cells, suggesting that pyrvinium pamoate is a promising agent that may be used for treatment of patients with imatinib-resistant T315I BCR-ABL CML in addition to imatinib-sensitive wild-type CML cells.

\section{Acknowledgements}

The present study was supported by grants from the National Natural Science Foundation of China (grant nos. 81025021, U1301226 and 81373434 to Professor J.P.; grant nos. 81473247 and 81673451 to Dr Y.J.), Guangdong Natural Science Funds for Distinguished Young Scholars (grant no. 2016A030306036 to Dr Y.J.), the Natural Science Foundation of Guangdong Province (grant no. 2015A030312014 to Professor J.P.), and the Research Foundation of Education Bureau of Guangdong Province, China (grant no. cxzd1103 to Professor J.P.). 


\section{References}

1. Rowley JD: Letter: A new consistent chromosomal abnormality in chronic myelogenous leukaemia identified by quinacrine fluorescence and Giemsa staining. Nature 243: 290-293, 1973.

2. McLaughlin J, Chianese $\mathrm{E}$ and Witte $\mathrm{ON}$ : In vitro transformation of immature hematopoietic cells by the P210 BCR/ABL oncogene product of the philadelphia chromosome. Proc Natl Acad Sci USA 84: 6558-6562, 1987.

3. Slupianek A, Falinski R, Znojek P, Stoklosa T, Flis S, Doneddu V, Pytel D, Synowiec E, Blasiak J, Bellacosa A and Skorski T: BCR-ABL1 kinase inhibits uracil DNA glycosylase UNG2 to enhance oxidative DNA damage and stimulate genomic instability. Leukemia 27: 629-634, 2013.

4. Kim SJ, Jung KH, Yan HH, Son MK, Fang Z, Ryu YL, Lee H, Lim JH, Suh JK, Kim J, et al: HS-543 induces apoptosis of Imatinib-resistant chronic myelogenous leukemia with T315I mutation. Oncotarget 6: 1507-1518, 2015.

5. Bu Q, Cui L, Li J, Du X, Zou W, Ding K and Pan J: SAHA and S116836, a novel tyrosine kinase inhibitor, synergistically induce apoptosis in imatinib-resistant chronic myelogenous leukemia cells. Cancer Biol Ther 15: 951-962, 2014.

6. Senior M: FDA halts then allows sales of Ariad's leukemia medication. Nat Biotechnol 32: 9-11, 2014.

7. Jung RC: Treatment of intestinal parasitic disease. South Med J 69: 799-804, 1976.

8. Lamb R, Ozsvari B, Lisanti CL, Tanowitz HB, Howell A, Martinez-Outschoorn UE, Sotgia F and Lisanti MP: Antibiotics that target mitochondria effectively eradicate cancer stem cells, across multiple tumor types: Treating cancer like an infectious disease. Oncotarget 6: 4569-4584, 2015.

9. Xu W, Lacerda L, Debeb BG, Atkinson RL, Solley TN, Li L, Orton D, McMurray JS, Hang BI, Lee E, et al: The antihelmintic drug pyrvinium pamoate targets aggressive breast cancer. PLoS One 8: e71508, 2013.

10. Deng L, Lei Y, Liu R, Li J, Yuan K, Li Y, Chen Y, Liu Y, Lu Y, Edwards CK III, et al: Pyrvinium targets autophagy addiction to promote cancer cell death. Cell Death Dis 4: e614, 2013.

11. Harada Y, Ishii I, Hatake K and Kasahara T: Pyrvinium pamoate inhibits proliferation of myeloma/erythroleukemia cells by suppressing mitochondrial respiratory complex I and STAT3. Cancer Lett 319: 83-88, 2012.

12. Yu DH, Macdonald J, Liu G, Lee AS, Ly M, Davis T, Ke N, Zhou D, Wong-Staal F and Li QX: Pyrvinium targets the unfolded protein response to hypoglycemia and its anti-tumor activity is enhanced by combination therapy. PLoS One 3: e3951, 2008.

13. Thorne CA, Hanson AJ, Schneider J, Tahinci E, Orton D, Cselenyi CS, Jernigan KK, Meyers KC, Hang BI, Waterson AG, et al: Small-molecule inhibition of Wnt signaling through activation of casein kinase $1 \alpha$. Nat Chem Biol 6: 829-836, 2010.

14. Li B, Fei DL, Flaveny CA, Dahmane N, Baubet V, Wang Z, Bai F, Pei XH, Rodriguez-Blanco J, Hang B, et al: Pyrvinium attenuates Hedgehog signaling downstream of smoothened. Cancer Res 74: 4811-4821, 2014

15. Li B, Flaveny CA, Giambelli C, Fei DL, Han L, Hang BI, Bai F, Pei XH, Nose V, Burlingame O, et al: Repurposing the FDA-approved pinworm drug pyrvinium as a novel chemotherapeutic agent for intestinal polyposis. PLoS One 9: e101969, 2014.

16. Xiang W, Cheong JK, Ang SH, Teo B, Xu P, Asari K, Sun WT, Than H, Bunte RM, Virshup DM and Chuah C: Pyrvinium selectively targets blast phase-chronic myeloid leukemia through inhibition of mitochondrial respiration. Oncotarget 6: 33769-33780, 2015 .
17. Zhang H, Trachootham D, Lu W, Carew J, Giles FJ, Keating MJ, Arlinghaus RB and Huang P: Effective killing of Gleevec-resistant CML cells with T315I mutation by a natural compound PEITC through redox-mediated mechanism. Leukemia 22: 1191-1199, 2008.

18. Lu Z, Jin Y, Qiu L, Lai Y and Pan J: Celastrol, a novel HSP90 inhibitor, depletes Bcr-Abl and induces apoptosis in imatinib-resistant chronic myelogenous leukemia cells harboring T315I mutation. Cancer Lett 290: 182-191, 2010.

19. Qiu L, Wang XD, Yu BH, Lu RZ, Ge F, Wang XL, Chen LJ, Han BH, Zhan ZM, Zhang BL and Ma J: Effect of a novel tyrosine kinase inhibitor HHGV678 on growth inhibition of Bcr-Abl wild type and IM-resistant cell lines in vitro. Zhongguo Shi Yan Xue Ye Xue Za Zhi 16: 1039-1043, 2008 (In Chinese).

20. Pan J, Quintas-Cardama A, Kantarjian HM, Akin C, Manshouri T, Lamb P, Cortes JE, Tefferi A, Giles FJ and Verstovsek S: EXEL-0862, a novel tyrosine kinase inhibitor, induces apoptosis in vitro and ex vivo in human mast cells expressing the KIT D816V mutation. Blood 109: 315-322, 2007.

21. Jin Y, Chen Q, Shi X, Lu Z, Cheng C, Lai Y, Zheng Q and Pan J: Activity of triptolide against human mast cells harboring the kinase domain mutant KIT. Cancer Sci 100: 1335-1343, 2009.

22. Jin Y, Lu Z, Ding K, Li J, Du X, Chen C, Sun X, Wu Y, Zhou J and Pan J: Antineoplastic mechanisms of niclosamide in acute myelogenous leukemia stem cells: Inactivation of the NF-kappaB pathway and generation of reactive oxygen species. Cancer Res 70: 2516-2527, 2010.

23. Lu B, Li J,Pan J,Huang B,Liu J and Zheng D: Everolimus enhances the cytotoxicity of bendamustine in multiple myeloma cells through a network of pro-apoptotic and cell-cycle-progression regulatory proteins. Acta Biochim Biophys Sin (Shanghai) 45: 683-691, 2013.

24. Shi X, Jin Y, Cheng C, Zhang H, Zou W, Zheng Q, Lu Z, Chen Q, Lai Y and Pan J: Triptolide inhibits Bcr-Abl transcription and induces apoptosis in STI571-resistant chronic myelogenous leukemia cells harboring T315I mutation. Clin Cancer Res 15: 1686-1697, 2009.

25. Pan J, Xu G and Yeung SC: Cytochrome $c$ release is upstream to activation of caspase- 9 , caspase- 8 , and caspase- 3 in the enhanced apoptosis of anaplastic thyroid cancer cells induced by manumycin and paclitaxel. J Clin Endocrinol Metab 86: 4731-4740, 2001.

26. Blonska M, Shambharkar PB, Kobayashi M, Zhang D, Sakurai H, Su B and Lin X: TAK1 is recruited to the tumor necrosis factor-alpha (TNF-alpha) receptor 1 complex in a receptor-interacting protein (RIP)-dependent manner and cooperates with MEKK 3 leading to NF-kappaB activation. J Biol Chem 280: 43056-43063, 2005.

27. Zhang X, Lou Y, Zheng X, Wang H, Sun J, Dong Q and Han B: Wnt blockers inhibit the proliferation of lung cancer stem cells. Drug Des Devel Ther 9: 2399-2407, 2015.

28. Beck JW, Saavedra D, Antell GJ and Tejeiro B: The treatment of pinworm infections in humans (enterobiasis) with pyrvinium chloride and pyrvinium pamoate. Am J Trop Med Hyg 8: 349-352, 1959.

29. Liu MD, Xiong SJ, Tan F and Liu Y: Physcion 8-O- $\beta$-glucopyranoside induces mitochondria-dependent apoptosis of human oral squamous cell carcinoma cells via suppressing survivin expression. Acta Pharmacol Sin 37: 687-697, 2016.

30. Basu D, Reyes-Mugica M and Rebbaa A: Role of the beta catenin destruction complex in mediating chemotherapy-induced senescence-associated secretory phenotype. PLoS One 7: e52188, 2012. 